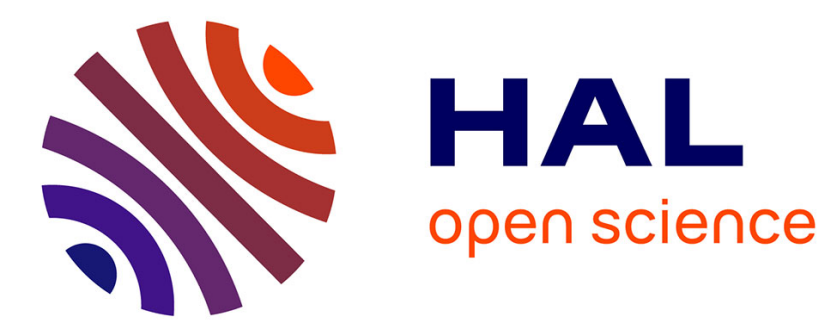

\title{
Side Payments as Barriers to Entry in Non-Neutral Networks
}

\author{
Bruno Tuffin
}

\section{To cite this version:}

Bruno Tuffin. Side Payments as Barriers to Entry in Non-Neutral Networks. 12th Conference of Telecommunication, Media and Internet Techno-Economics (CTTE), Nov 2015, Munich, Germany. hal-01149214v2

\section{HAL Id: hal-01149214 \\ https://hal.inria.fr/hal-01149214v2}

Submitted on 8 Sep 2015

HAL is a multi-disciplinary open access archive for the deposit and dissemination of scientific research documents, whether they are published or not. The documents may come from teaching and research institutions in France or abroad, or from public or private research centers.
L'archive ouverte pluridisciplinaire HAL, est destinée au dépôt et à la diffusion de documents scientifiques de niveau recherche, publiés ou non, émanant des établissements d'enseignement et de recherche français ou étrangers, des laboratoires publics ou privés. 


\title{
Side Payments as Barriers to Entry in Non-Neutral Networks
}

\author{
Bruno Tuffin \\ Inria, France \\ Email: bruno.tuffin@inria.fr
}

\begin{abstract}
In the network neutrality debate, content providers fight against side payments imposed by Internet Service Providers, arguing that it could slow down innovation. But at the same time some big providers actually pay those fees while still officially in favor of neutrality. To better understand this strategical behavior, this paper proposes a simple model providing some insight on whether or not paying side payments for an incumbent provider is a way to create barriers to entry for competitors. It also investigates the economic consequences on all actors: incumbent and new entrant content providers, users, and the Internet Service Provider. We then describe how the side payment can be determined as a Nash bargaining solution.
\end{abstract}

\section{INTRODUCTION}

The network neutrality debate is shaking up not only the telecommunication world but also countries governments with laws passed worldwide. This vivid debate comes from Internet Service Providers (ISPs) complaining about some distant but heavy resource-consuming Content Providers (CPs) creating stress on their network while not paying anything to them because connected to the Internet through another ISP. They therefore threat to block or slow down their traffic if they do not pay a side fee in order to participate in the network infrastructure maintenance and upgrade. This created a lot of protests from user associations and content providers claiming that it could harm innovation, freedom of speech, and the universality of access principle among other issues. The issue is therefore basically whether the network should forbid differentiation or not. For more information about the history of the network neutrality debate, and arguments from both sides, the reader is advised to go to [1]-[4] among the numerous publications on the topic.

Surprisingly, some big CPs, while officially strongly in favor of neutrality, are now giving side payments to ISPs. This is typically what Google (a major CP because of YouTube traffic) is doing, for instance in France with the main ISP Orange ${ }^{1}$, Netflix with Comcast and Verizon ${ }^{2}$, etc.

A question is then: what reason drives big CPs to accept such side payments, when governments and user associations seem to fight on their side? In this paper, we investigate

\footnotetext{
$1_{\text {http://www.theverge.com/2013/1/19/3894182/french-isp-orange- says-google-pays-to-send-traffic }}$ 2 http://arstechnica.com/information-technology/2014/05/21/see-which-isps-google-microsoft-and-netflix-tradeinternet-traffic-with/
}

the relevance of a potential reason: accepting side payments when able to pay them could create a barrier to entry for potential competitors. Indeed, new entrants may incur initial costs and not have the same economies of scale than incumbents; therefore they may not be able to afford such payments. On the other hand, the cost of side payments for incumbent providers may be overcome by the potential loss due to competitors entering the market.

Barrier to entry definition has been a topic of discussion in the economic literature. It has first been defined by Bain in 1956 as "anything that allows incumbent firms to earn above-normal profits without the treat of entry" [5]. Other definitions followed. A historical development of definitions and a classification was provided in [6] with four different concepts of barrier to entry: ii) an economic barrier to entry as a cost incurred by a new entrant but not by an incumbent, ii) an antitrust barrier to entry as a cost delaying entry and as a consequence reducing social welfare compared to an immediate cost, iii) a primary barrier to entry as a cost that constitutes a barrier to entry on its own, and iv) an ancillary barrier to entry as a cost that (indirectly) reinforces other barriers to entry. A side payment falls into the third category (primary barrier to entry) since directly incurred by CPs and potentially preventing market entrance if benefits are not sufficient.

We propose in this paper to introduce and analyze a model describing a simple Internet supply chain with two content providers, end users, and ISPs as intermediaries. Demand from users will be assumed to depend on the access price at the ISP, and to follow a (traditional) linear form. We will analyze several scenarios:

- without side payment (a neutral network) and with competition between ISPs,

- with side payments and competition,

- and with side payments and only the incumbent CP.

We will compare the outputs obtained in theses scenarios. We will particularly look at revenues of CPs and the ISP, and consumer surplus. The goal is to get some insight about what strategy each actors should choose and whether or not some regulation rules should be imposed. If collusion between the incumbent CP and the ISP is beneficial, we will 
determine the side payment as a Nash bargaining solution [7], an axiomatic concept describing a solution of negotiation between players.

Note that modeling and analysis of barrier to entry in markets has of course been already studied, in a spirit somewhat similar to our work. A. Dixit in his seminal work [8] considers also a direct competition between an incumbent and an entrant providers, with linear demand too, but using a model less representative of the Internet without ISPs as intermediaries, whereas ISPs make here important decisions. [9] is another work making use of a basic model, but working on a different issue: determining the optimal number of firms in a market depending on fixed costs.

The rest of this paper is organized as follows. In Section II, the mathematical model is presented, as well as the order of decision among players, those playing first strategically anticipating the subsequent decisions of the others. Section III then provides the resulting values of revenues and consumer surplus in the three scenarios: no side payment, side payment but only the incumbent $\mathrm{CP}$, and side payments with the two CPs. Those outputs are compared and conditions under which creating barriers to entry are beneficial for the incumbent provider are provided. Section IV then describes how the incumbent $\mathrm{CP}$ and the ISP can negotiate the price, defined as the Nash bargaining solution. Finally Section V concludes our paper and discusses extensions of the model.

\section{Model}

We consider the model presented in Figure 1. It describes a supply chain made of two content providers (CPs) in competition, end users, and one ISP as an intermediary. We just consider two CPs, believing that it is to provide some insight (which is the goal of the paper), but this can be generalized and will be the purpose of future work. To simplify the analysis, users are treated as a continuum, meaning that no user has individually an impact on the system (i.e., they are assumed infinitesimal). The total mass of base users is defined as $D$.

Figure 1 also shows the economic relations between actors: there is potentially a cost $c$ (side payment) per unit of volume that CPs transfer to the ISP, and a subscription fee $p$ per unit of customer paid by customers to the ISP. We also assumer a revenue $r_{i}$ (respectively $r_{e}$ ) per unit of customer for the incumbent $\mathrm{CP}$ (respectively the entrant $\mathrm{CP}$ ). Those revenues may for instance be due to advertisements displayed on the $\mathrm{CP}$ site and seen or clicked by customers, but could apply to other types of revenue (sales, subscriptions, etc.).

\section{A. Users}

Demand (mass of users) $d$ is assumed to be linear in price,

$$
d=(D-\alpha p)^{+}
$$

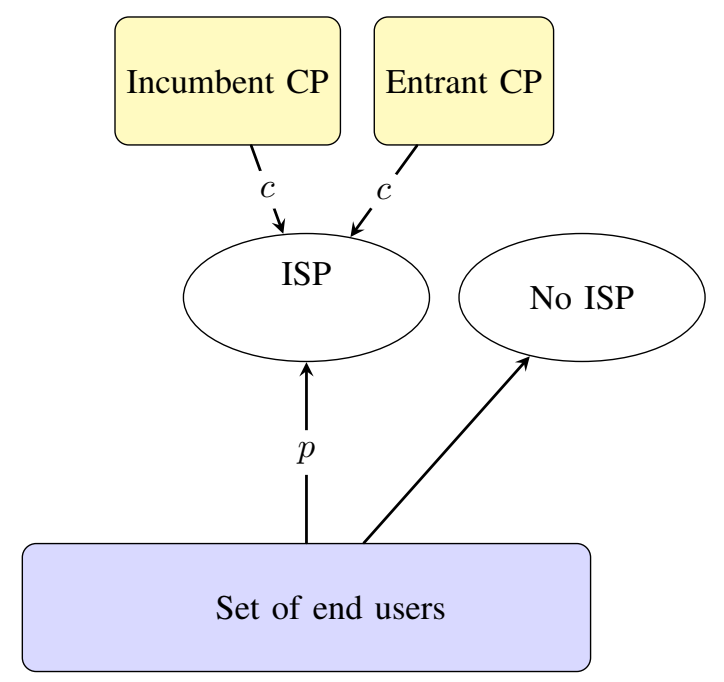

Figure 1. Representation of relations between users, ISP and CPs

where $\alpha$ is the price sensitivity parameter and $(\cdot)^{+}=$ $\max (\cdot, 0)$. This type of model is traditional in economics [7] and has often been used in models studying barriers to entry such as [8].

We also decompose the total demand $d$ into $\mathrm{d}_{i}$, the mass of users making use of the service of the incumbent and $d_{e}$ the mass of users making use of the new entrant service. In the case where the incumbent is alone, we have $d_{i}=d$ and $d_{e}=0$. On the other hand when both propose their services, $d_{i}=\gamma_{i} d$ and $d_{e}=\gamma_{e} d$ with $\gamma_{i}, \gamma_{e} \in(0,1]$. We will assume $\gamma_{i}+\gamma_{e} \geq 1$, meaning that users might use both services (there is no exclusivity) when they are both present.

Note here that demand does not depend on content, more exactly on whether or nor the two CPs are present or not. This can be justified by the fact that the CPs we are considering are just a part of the Internet content and having one or two substitutable CPs does not limit users range of activities (or has a negligible impact).

A metric of interest as a measure of consumer satisfaction from the users' side is Consumer Surplus (CS). Recall the definition of (aggregated) CS: it is the aggregated difference between the maximum price a consumer is willing to pay and the actual paid price. Mathematically, it is the hashed area in Figure 2.

\section{B. Internet Service Provider}

The ISP s characterized by its revenue

$$
R=p d+c v_{i}+c v_{e}
$$

where

- $v_{i}$ and $v_{e}$ are the corresponding units of volume with

$$
v_{i}=\beta_{i} d_{i} \quad \text { and } \quad v_{e}=\beta_{e} d_{e}
$$




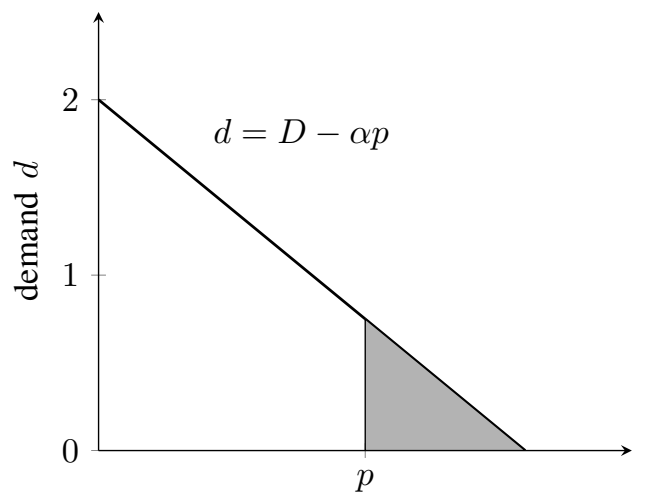

Figure 2. Consumer surplus with a linear demand.

with $\beta_{i}$ and $\beta_{e}$ the (average) units of volume consumed per user;

- $p d$ corresponds to the revenue due to subscriptions from users.

\section{Content Providers}

Recall that we have defined $d_{i}$ and $d_{e}$ as the masses of users of the incumbent and new entrant CPs respectively, and $r_{i}$ and $r_{e}$ the respective revenues generated per (mass unit of) customer.

The revenues of CPs are then:

$$
\begin{aligned}
& R_{i}=r_{i} d_{i}-c v_{i}=\left(r_{i}-\beta_{i} c\right) d_{i} \\
& R_{e}=\left(r_{e}-\beta_{e} c\right) d_{e} .
\end{aligned}
$$

\section{Order of decisions}

CPs, ISP and users have to take strategical decisions, but they are not all taken at the same time scale. The decisions are as follows:

1) First, the ISP and incumbent CP decide (through a negotiation) the side payment;

2) Then the ISP decides the subscription price $p$;

3) Finally users adapt their demand to the proposed services and price.

Decisions are taken strategically, by backward induction: even when playing first, actors will make their decision anticipating what will be the subsequent decisions of the others.

This model is studied in the next section in the case of three scenarios:

1) no side payments and both CPs in the market;

2) side payments and only the incumbent provider;

3) side payments and both CPs.

The interest of considering those three scenarios, and comparing them, is many folds: First, it will help the incumbent provider to decide whether or not to be in favor of side payments and their level of magnitude, by comparing the revenues to the cases where the new entrant is there. Second, it helps the ISP to decide a strategy on side payments by looking at its revenue, and also if negotiating with the incumbent is beneficial as opposed to the threat of network neutrality regulation. Finally, the same comparison is helpful for regulatory bodies to investigate if it harms competition and consumer surplus.

Note that in all cases, the subscription price is chosen to maximize the ISP revenue

$$
R=p d+\left(c \beta_{i} \gamma_{i}+c \beta_{e} \gamma_{e}\right) d
$$

where we set $\gamma_{e}=0$ and $\gamma_{i}=1$ if the incumbent is alone. To simplify the notations, define $C=c\left(\beta_{i} \gamma_{i}+\beta_{e} \gamma_{e}\right)$ as the average side payment per (unit of) user. From $R=(p+$ $C)(D-\alpha p)$, we get

$$
p=\frac{D}{2 \alpha}-\frac{C}{2}
$$

as the price maximizing the ISP revenue.

Remark that this price could be negative if $C>D / \alpha$ but this would mean a demand larger than the base $D$ (in the expression of demand, we should have set $D$ as a max). Taking $p=0$ is then optimal.

\section{OUTPUTS FOR GIVEN SIDE PAYMENTS}

This section describes the outputs for the three scenarios and then deduces the strategic decisions of players.

\section{A. Without side payments}

It amounts to assume $c=0$.

Replacing this value of $c$ in the price optimization and substituting the optimal price and corresponding demand value in the expressions of revenues and CS, we get

$$
\begin{aligned}
p & =\frac{D}{2 \alpha} \\
d & =\frac{D}{2} \\
R_{i} & =r_{i} \gamma_{i} \frac{D}{2} \\
R_{e} & =r_{e} \gamma_{e} \frac{D}{2} \\
R & =\frac{D^{2}}{4 \alpha} \\
C S & =\int_{\frac{D}{2 \alpha}}^{\frac{D}{\alpha}}(D-\alpha p) d p=\frac{D^{2}}{8 \alpha} .
\end{aligned}
$$

Recall that the expression of CS is the hashed area in Figure 2. 
B. With side payments but only the incumbent content provider

Then $\gamma_{i}=1, \gamma_{e}=0$, and $C=c \beta_{i}$. For a given side payment $c$, we get, following the same substitution procedure than in previous subsection,

$$
\begin{aligned}
p & =\frac{D}{2 \alpha}-\frac{c \beta_{i}}{2} \text { (assumed non-negative) } \\
d=d_{i} & =\frac{D}{2}+\alpha \frac{c \beta_{i}}{2} \\
R_{i} & =\left(r_{i}-\beta_{i} c\right)\left(\frac{D}{2}+\alpha \frac{c \beta_{i}}{2}\right) \\
R & =\frac{1}{4 \alpha}\left(D+\alpha c \beta_{i}\right)^{2} \\
C S & =\int_{\frac{D}{2 \alpha}-\frac{c \beta_{i}}{2}}^{\frac{D}{\alpha}}(D-\alpha p) d p \\
& =\frac{D^{2}+2 D c \beta_{i} \alpha+c^{2} \beta_{i}^{2} \alpha^{2}}{8 \alpha} .
\end{aligned}
$$

C. With side payments and the two content providers

It gives in this case

$$
\begin{aligned}
p & =\frac{D}{2 \alpha}-\frac{c\left(\beta_{i} \gamma_{i}+\beta_{e} \gamma_{e}\right)}{2} \\
d & =\frac{D}{2}+\alpha \frac{c\left(\beta_{i} \gamma_{i}+\beta_{e} \gamma_{e}\right)}{2} \\
R_{i} & =\gamma_{i}\left(r_{i}-c \beta_{i}\right)\left(\frac{D}{2}+\alpha \frac{c\left(\beta_{i} \gamma_{i}+\beta_{e} \gamma_{e}\right)}{2}\right) \\
R_{e} & =\gamma_{e}\left(r_{e}-c \beta_{e}\right)\left(\frac{D}{2}+\alpha \frac{c\left(\beta_{i} \gamma_{i}+\beta_{e} \gamma_{e}\right)}{2}\right) \\
R & =\frac{1}{4 \alpha}\left(D+\alpha c\left(\beta_{i} \gamma_{i}+\beta_{e} \gamma_{e}\right)\right)^{2} \\
C S & =\frac{D^{2}+2 D c\left(\beta_{i} \gamma_{i}+\beta_{e} \gamma_{e}\right) \alpha+c\left(\beta_{i} \gamma_{i}+\beta_{e} \gamma_{e}\right)^{2} \alpha^{2}}{8 \alpha} .
\end{aligned}
$$

\section{Comparison and resulting strategic decisions}

We can make several remarks when comparing the outputs for the three scenarios:

1) First, the new entrant will not enter the market if its revenue is negative, i.e., if $c>r_{e} / \beta_{e}$. The incumbent $\mathrm{CP}$, if wishing to prevent the new entrant to reach customers, must agree on side payments of at least that amount. Clearly also, the incumbent cannot accept a non-negative revenue, hence we must have $c \leq r_{i} / \beta_{i}$. It implicitly means that the revenue per unit of data $r_{i} / \beta_{i}$ has to be larger for the incumbent than that $r_{e} / \beta_{e}$ of the new entrant. This will be assumed from now and is a reasonable assumption.

2) But in order for the incumbent to be in favor of side payments, and therefore to create barrier to entry, its revenue has also to be larger than i) when the new entrant is there (with side payments), and ii) when there is no side payments.
- First, the situation when it is beneficial to pay $c$ for the incumbent to and be alone in the market rather than paying and being in competition with the same $c$ is summarized by the equation (comparing the respective expressions of $R_{i}$ ):

$$
D+\alpha c \beta_{i}>\gamma_{i}\left(D+\alpha c\left(\beta_{i} \gamma_{i}+\beta_{e} \gamma_{e}\right)\right) .
$$

Note that for $\gamma_{i}$ small enough, this will always be true (that is, the new entrant make the incumbent lose too many clients; it is better to pay enough side payments to avoid its presence). But what amount $c$ of payment is required to be in favor of side payments? The above equation is equivalent to

$$
\left(1-\gamma_{i}\right) \frac{D}{\alpha}>c\left(\gamma_{i}\left(\beta_{i} \gamma_{i}+\beta_{e} \gamma_{e}\right)-\beta_{i}\right) .
$$

- If $\gamma_{i}\left(\beta_{i} \gamma_{i}+\beta_{e} \gamma_{e}\right)-\beta_{i} \leq 0$, this will always be true;

- If $\gamma_{i}\left(\beta_{i} \gamma_{i}+\beta_{e} \gamma_{e}\right)-\beta_{i}>0$, we need $c<((1-$ $\left.\left.\gamma_{i}\right) \frac{D}{\alpha}\right) /\left(\gamma_{i}\left(\beta_{i} \gamma_{i}+\beta_{e} \gamma_{e}\right)-\beta_{i}\right)$.

- In addition, being in monopoly with side payments has to be preferred to the (neutral) competitive case without fee. The former situation is preferred to the latter if $\left(r_{i}-\beta_{i} c\right)\left(D+\alpha c \beta_{i}\right)>r_{i} \gamma_{i} D$, i.e., if $c$ is in the interval

$$
\begin{aligned}
& \left(\frac{-\left(D-\alpha r_{i}\right)-\sqrt{\beta_{i}^{2}\left(D-\alpha r_{i}\right)^{2}+4 \alpha \beta_{i}^{2} r_{i}\left(1-\gamma_{i}\right) D}}{2 \alpha \beta_{i}^{2}},\right. \\
& \left.\frac{-\left(D-\alpha r_{i}\right)+\sqrt{\beta_{i}^{2}\left(D-\alpha r_{i}\right)^{2}+4 \alpha \beta_{i}^{2} r_{i}\left(1-\gamma_{i}\right) D}}{2 \alpha \beta_{i}^{2}}\right) .
\end{aligned}
$$

The left bound of the interval being negative, we actually just need $c$ to be smaller than the right bound.

Compiling those two results, the incumbent $\mathrm{CP}$ will clearly prefer paying the ISP for its traffic and create barriers to entry if

$$
\begin{aligned}
c< & \min \left(\left(\left(1-\gamma_{i}\right) \frac{D}{\alpha}\right) /\left(\gamma_{i}\left(\beta_{i} \gamma_{i}+\beta_{e} \gamma_{e}\right)-\beta_{i}\right),\right. \\
& \left.\frac{-\left(D-\alpha r_{i}\right)+\sqrt{\beta_{i}^{2}\left(D-\alpha r_{i}\right)^{2}+4 \alpha \beta_{i}^{2} r_{i}\left(1-\gamma_{i}\right) D}}{2 \alpha \beta_{i}^{2}}\right)
\end{aligned}
$$

if $\gamma_{i}\left(\beta_{i} \gamma_{i}+\beta_{e} \gamma_{e}\right)-\beta_{i}>0$, and just if

$$
c<\frac{-\left(D-\alpha r_{i}\right)+\sqrt{\beta_{i}^{2}\left(D-\alpha r_{i}\right)^{2}+4 \alpha \beta_{i}^{2} r_{i}\left(1-\gamma_{i}\right) D}}{2 \alpha \beta_{i}^{2}}
$$

if $\gamma_{i}\left(\beta_{i} \gamma_{i}+\beta_{e} \gamma_{e}\right)-\beta_{i} \leq 0$.

Remark that the incumbent can at the same time pay a level of fee to ensure that the new entrant will not be there and ask for no side payments publicly. This is typically what would happen if the revenue with side payment and barrier to entry is i) larger than that with the new entrant (and still a side payment), but ii) smaller than that with a "neutral" ISP. It corresponds to the situation when the $\mathrm{CP}$ would prefer the neutral 


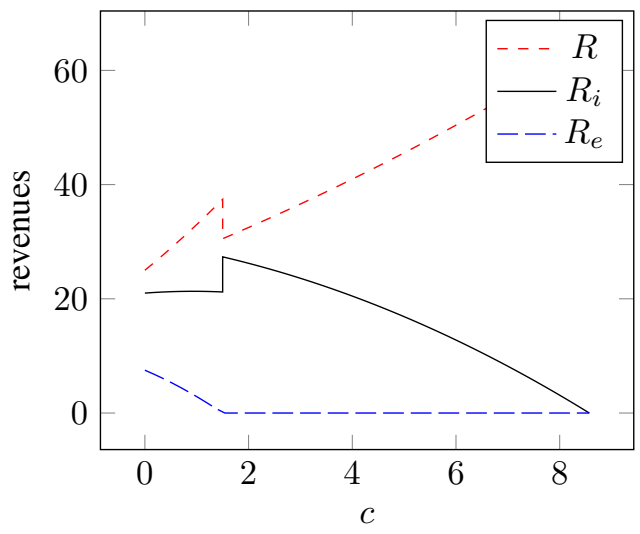

Figure 3. Revenues in terms of $c$.

behavior, but being unsure that it will be applied, goes to the best of two evils. It may also be strategical even if monopoly plus side payments is the best option, in terms of image and in order to better negotiate the level of payments (see next section). It may explain what Google is doing in France: paying a side payment to the major ISP (Orange), but asking for a neutral network.

3) The ISP has also to agree that the level of side payment increases its revenue $R$. This is always the case with respect to a neutral situation. But a single $\mathrm{CP}$ will be preferred if $c_{s} \beta_{i}>c_{2}\left(\beta_{i} \gamma_{i}+\beta_{e} \gamma_{e}\right)$ where $c_{s}$ is the side payment when there is only one CP (through a corresponding negotiation, and $c_{2}$ when there are two. If $c_{s}=c_{2}$, we just need $\beta_{i}>\beta_{i} \gamma_{i}+\beta_{e} \gamma_{e}$. But this inequality is true if the incumbent induces a large amount of traffic (typically what Google does with YouTube).

4) Consider now consumer surplus. If a regulator investigates the consequences of side payments, they are actually beneficial from a customer point of view. This comes from the fact that due to side payments, the ISP can reduce the access price, hence higher levels of demand and satisfaction from end users. This situation is actually at the expense of CPs instead and only.

Example 1. Figure 3 displays the revenues in terms of $c$ when $D=10, \alpha=1, \beta_{i}=0.7, \beta_{e}=2, r_{i}=6, r_{e}=3$, $\gamma_{i}=0.7$, and $\gamma_{e}=0.5$ (arbitrary values). We display the revenues $R_{i}$ and $R$ in the competitive case when $c<r_{e} / \beta_{e}$, and in the monopolistic case when $r_{e} / \beta_{e} \leq c<r_{i} / \beta_{i}$. We clearly see the threshold at $r_{e} / \beta_{e}=1.5$ where the new $C P$ strategy changes from entering the market to not entering it. We also see that the incumbent $C P$ is better off with a side payment when above but close to that threshold.

Table I presents some numerical computations for the three scenarios, for two values of $c$. If the price is $c=0.5$, the incumbent $C P$ even gets a higher revenue than in the two other cases when alone, but with such a c, the new entrant would be present; we would be in the situation of side payment and competition. On the other hand, we see that with a price $c=2$ the new entrant should not enter the market, and the incumbent gets a higher revenue than without side payment or with a side payment smaller than $r_{e} / \beta_{e}=1.5$ (something clear on Figure 3 ). The revenue of the ISP is also higher than when no side payment, hence is better solution for both the ISP and incumbent CP, and therefore an incentive to cooperate. In terms of consumer surplus, the higher the side payment, the better it is.

\section{SidE PAYMENTS NEGOTIATION}

In the previous section, we were discussing the optimal strategies when the side payment was fixed. The purpose of this section is to investigate how those payments can be defined, if they are eventually authorized by regulators. Actually several possibilities exist. A first one would be that the price is chosen by the ISP trying to maximize its revenue. It is clear from the revenue expressions that it would be set at a level such that the $\mathrm{CP}$ revenue is becoming 0 , at the same time increasing demand because most ISP revenue is then paid by the CP. In such a case, there would a strong opposition and lobbying from CPs, and the risk of neutrality regulation would be very large.

We consider here the situation where there is a negotiation between the incumbent $\mathrm{CP}$ and the ISP about the side payment. The solution of this negotiation is assumed to be the result of an axiomatic model called Nash bargaining solution [10]. Placed into our context, the ISP and incumbent $\mathrm{CP}$ independently choose a set of acceptable side payments, the ones providing a non-negative revenue in the interval $\left(r_{e} / \beta_{e}, r_{i} / \beta_{i}\right)$ ensuring for the incumbent to impose barriers to entry. If there is no agreement, a threat is executed: due to eventual complains related to the network neutrality debate, the threat is no side payment, $c=0$. Since this negotiation may end up with several equilibria, the most likely to be played is the one maximizing the product of the utilities minus the utility at the threat [11]. In other words, that equilibrium side payment $c$ maximizes

$$
K=\left(R_{i}(c)-R_{i}(0)\right)(R(c)-R(0)),
$$

where $R_{i}(c)$ and $R(c)$ are the revenues with side payment $c$, and that maximizer is called the Nash bargaining solution.

Since $c>r_{e} / \beta_{e}$, only the incumbent $\mathrm{CP}$ is present and

$$
K=\left(\left(r_{i}-\beta_{i} c\right)\left(\frac{D}{2}+\alpha \frac{c \beta_{i}}{2}\right)-r_{i} \gamma_{i} \frac{D}{2}\right) \frac{c \beta_{i}}{4}\left(c \alpha \beta_{i}+2 D\right) \text {. }
$$

We can differentiate this term with respect to $c$ and get optimum values (that will be summarized to solving polynoms in $c$ of degree three). Instead of providing this analytic list which does not bring much insight, we illustrate on our running example the Nash bargaining solution for $c$.

Example 2. Figure 4 displays $K$ in terms of $c$ (still when $D=10, \alpha=1, \beta_{i}=0.7, \beta_{e}=2, r_{i}=6, r_{e}=3, \gamma_{i}=0.7$, 


\begin{tabular}{|c|c|c|c|c|c|c|c|}
\hline$c$ & Scenario & $p$ & $d$ & $R_{i}$ & $R_{e}$ & $R$ & $C S$ \\
\hline- & No side payment & 5 & 5 & 21 & 7.5 & 25 & 12.5 \\
\hline 0.5 & Monopoly & 4.825 & 5.175 & 29.23875 & - & 26.780625 & 13.3903125 \\
\hline 0.5 & Side payment and competition & 4.6275 & 5.3725 & 21.2482375 & 5.3725 & 28.86375625 & 14.43187812 \\
\hline 2 & Monopoly & 4.3 & 5.7 & 26.22 & - & 32.49 & 16.245 \\
\hline 2 & Side payment and competition & 3.51 & 6.49 & 20.8978 & -3.245 & 42.1201 & 21.06005 \\
\hline
\end{tabular}

SOME NUMERICAL VALUES FOR THE THREE SCENARIOS FOR TWO VALUES OF $c$

\begin{tabular}{c|c|c|c|c|c|c|c|}
$c$ & Scenario & $p$ & $d$ & $R_{i}$ & $R_{e}$ & $R$ & $C S$ \\
\hline \hline 2.137012 & Nash Bargaining & 4.2520458 & 5.7479542 & 25.88931223 & - & 33.03897748 & 16.51948874
\end{tabular}

NUMERICAL VALUES FOR $c$ AS THE NASH BARGAINING SOLUTION.

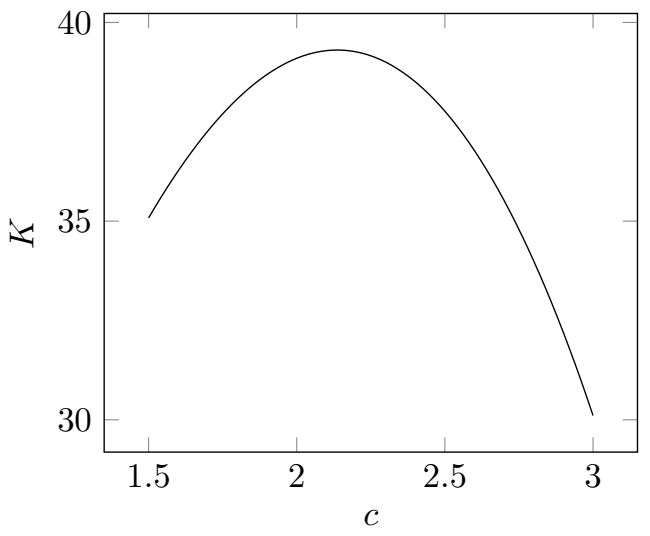

Figure 4. $K$ in terms of $c$.

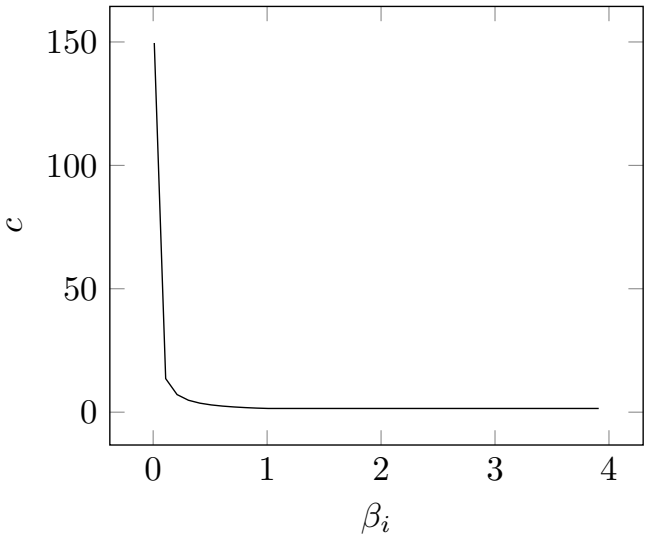

Figure 5. Nash bargaining $c$ in terms of $\beta_{i}$. and $\left.\gamma_{e}=0.5\right)$. One can check that, with our parameters, $K$ is maximized at $c=2.137012$. Table II presents the output at this value. The incumbent $C P$ revenue is not maximized (because smaller than when $c=2$ ), but much larger than when there is no side payment. The same applies for the ISP. It is indeed a compromise on which they both agree.

We also display in Figure 5 Nash bargaining $c$ in terms of $\beta_{i}$ when the other parameters are defined as above. This side payment $c$ is larger than the threshold $r_{e} / \beta_{e}=1.5$ for "small" values of $\beta_{i}$, and sticks to that threshold as soon as $\beta_{i} \geq 1.0$.

\section{CONCLUSions}

In this paper, we have introduced a simple model representing the Internet supply chain and analyzed the relevance for incumbent CPs to agree to pay side payments (depending on parameter values) to ISPs in order to introduce barrier to entry for competitors. This helps to understand the current behavior of some big CPs, paying those fees despite militating against non-neutral networks. We believe that even if simple, the presented model provides some insight on the consequence on all categories of actors.

In terms of future work, one assumption we would like to relax is the known parameter values for the new entrant. Indeed, it is not easy to determine before negotiating the side payment what would be the new entrant provider's revenue, market share, etc. We would like to replace those deterministic values by known distributions (or beliefs based on experience) and then try to maximize expected revenues.

\section{ACKNOWLEDGEMENTS}

The author would like to thank the anonymous referees for suggestions that greatly improved the quality of the paper.

\section{REFERENCES}

[1] P. Crocioni, "Net neutrality in Europe: Desperately seeking a market failure," Telecommunications Policy, vol. 35, no. 1, pp. 1-11, 2011. [Online]. Available: http://www.sciencedirect.com/science/article/pii/ S0308596110001461

[2] P. Maillé, P. Reichl, and B. Tuffin, "Internet governance and economics of network neutrality," in Telecom. Eco. - Selected Results of the COST Action IS605 EcontTel, A. Hadjiantonis and B. Stiller, Eds. Springer Verlag, 2012, pp. 108-116.

[3] T. Lenard and R. May (Eds.), Net Neutrality or Net Neutering: Should Broadband Internet Services be Regulated. Springer, 2006.

[4] T. Wu, "Network neutrality, broadband discrimination," Journal of Telecom. and High Tech., 2003.

[5] J. Bain, Barriers to New Competition. Cambridge, MA: Harvard University Press, 1956. 
[6] R. P. Mcafee, H. M. Mialon, and M. A. Williams, "Economics and antitrust barriers to entry," 2003, http://vita.mcafee.cc/PDF/ Barriers2Entry.pdf.

[7] P. Maillé and B. Tuffin, Telecommunication Network Economics: From Theory to Applications. Cambridge University Press, 2014.

[8] A. Dixit, "A model of duopoly suggesting a theory of entry barriers," Bell Journal of Economics, vol. 10, no. 1, pp. 20-32, 1979.

[9] D. Heger and K. Kraft, "Barriers to entry and profitability," ZEW Zentrum für Europäische Wirtschaftsforschung / Center for European Economic Research, ZEW Discussion Papers 08-071, 2008. [Online]. Available: http://EconPapers.repec.org/RePEc:zbw:zewdip:7414

[10] M. Osborne and A. Rubinstein, A Course in Game theory. MIT Press, 1994

[11] J. Nash, "Two-person cooperative games," Econometrica: Journal of the Econometric Society, pp. 128-140, 1953. 